\title{
Towards a dynamical approach to the calculation of the figure of merit of thermoelectric nanoscale devices
}

\author{
Roberto D’Agosta, $, a, \ddagger$
}

'Research on thermoelectrical energy conversion, the reuse of waste heat produced by some mechanical or chemical processes to generate electricity, has recently gained some momentum. The calculation of the electronic parameters entering the figure 'of merit of this energy conversion, and therefore the discovery of efficient materials, is usually performed starting from the Landauer's approach to quantum transport coupled with the Onsager's linear response theory. As it is well known, that approach 'suffers of certain serious drawbacks. Here, we discuss alternative dynamical methods that can go beyond the validity of the Landauer's/Onsager's approach for electronic transport. They can be used to validate the predictions of the Landauer's/Onsager's 'approach and to investigate systems for which that approach has shown to be unsatisfactory.

\section{Introduction}

'Electrical energy is one of the most versatile forms of energy, 'since it can be easily stored, moved, or converted to any other form (work, heat, etc.). On the other hand, in the process of electrical energy production, a relevant part of the energy, in'deed as much as $60 \%$ in most cases, is wasted as heat, e.g., the plumes from the cooling towers of a power station. The "efficiency of this conversion process is limited by fundamental laws and, nonetheless, it is difficult to do much better than the present values. Nonetheless, no physical principle forbids us from using part of the waste heat of the first energy conversion to obtain more energy. Indeed, if the conversion process were ideally efficient no energy could be obtained through any other physical or chemical process. In reality, for non ideal cycles, by reusing the waste heat, we will pay a price in wasted energy, but looking at the overall process we will end up producing more electrical energy and less waste heat. This two-step pro'cess is limited in efficiency by thermodynamical laws, but it is still more efficient than the single step process. Many alternative methods to convert that waste heat back to energy have been proposed. We will focus on one of them, i.e., thermoelectricity, the direct conversion of a temperature gradient into an electrical current. $1-5$

Nanoscale devices have the power to revolutionise the field of electronics. ${ }^{6-9}$ It is clear that their comprehensive and exhaustive experimental exploration not driven by reliable theoretical predictions will be the result of serendipity not of scientific research. In the last few decades, Numerical and analytical tools have been developed to help understanding and designing efficient molecular devices. Remarkable successes have been scored, but the same tools for nearly all the most promising

${ }^{a}$ ETSF Scientific Development Center, Departamento de Física de Materiales, Universidad del País Vasco, E-20018 San Sebastián, Spain

${ }^{b}$ IKERBASQUE, Basque Foundation for Science, E-48011, Bilbao, Spain

$\ddagger$ Email: roberto.dagosta@ehu.es molecules, e.g., oxides for photovoltaic devices, have given mixed results. The general consensus goes in the direction of investigating the same devices with dynamical methods. 10 These allow a careful description of the dynamics of both the electronic and atomic parts of the device thereby understanding their interplay in establishing the electrical properties of the molecule. One aspect that is attracting increasing interest is how to make these molecular devices more stable. We know that thermal excitations either destabilise the chemical bonds that attach the molecule to the leads or effectively destroy the lead by breaking the metal-metal (usually $\mathrm{Au}$ ) chemical bonds of its components. 11 The investigation on how to efficiently remove the heat locally stored in the device is thus of primary importance towards a nanoscale electronics. 12 Here again, timedependent methods are the tools of choice, since they allow a fundamental description of the approach to a steady state ${ }^{13}-16$ (when a steady state is established) than the standard linear response theories. Finally, nanoscale devices seem to overcome some of the thermoelectric efficiency problems highlighted in bulk materials. A careful choice of the materials and an accurate tailoring of their properties may pave the way to efficient thermoelectric nanodevices.

The efficiency of the thermoelectric energy conversion is related to the so called figure of merit. This number is defined in terms of the microscopical quantities of the devices, i.e.,

$$
Z T=\frac{S^{2} \sigma}{\kappa} T
$$

where $S$ is the Seebeck coefficient, $\sigma$ the electrical conductance, $\kappa$ the thermal conductance, which includes the electronic and crystal contributions, and $T$ the operating temperature. The figure of merit determines how efficient the Peltier or Seebeck devices will be:

$$
\eta=\eta_{0} \frac{\sqrt{1+Z T}-1}{\sqrt{Z T+1}+T_{<} / T_{>}},
$$


where $\eta$ is the efficiency and $\eta_{0}$ is the efficiency of the Carnot ideal cycle, $\eta_{0}=\left(T_{>}-T_{<}\right) / T_{>}$. Here, $T_{>}$is the temperature of the hot junction, while $T_{<}$is the temperature of the cool junction. Typical values for $Z T$ in actual devices are around 1, and viable devices for technological applications need to have $Z T$ larger than 2-3.17

Nanomaterials have shown a plethora of interesting phenomena, many of which in the realm of electric and thermal transport ${ }^{8.9 .18}$. For application to the thermoelectric technology, certain nanomaterials like metal nanowires present the interesting feature that electrical and thermal transport are almost independent, in particular the electrons are mostly responsible for the electrical transport while lattice vibrations are mostly responsible for thermal transport. This allows us to tailor nanoscale devices in which the thermal conductance is minimised while keeping the electrical conductance constant. On the other hand, quantum mechanical effects can strongly modify the transport properties of nanomaterials. As discussed by Hicks and Dresselhaus, $\stackrel{19}{=}$ a key characteristic for determining the efficiency of the thermoelectric energy conversion is the electronic density of states of the device. In small strongly confined systems, a tailored density of states might induce a manyfold increase of the figure of merit. For this reason there is widespread belief that nanoscopic devices can show a large thermoelectric figure of merit. $\underline{19}$ There are other reasons for searching for efficient thermoelectric materials in sub-micron world. We have seen that for large energy production thermoelectric devices might prove not efficient enough. However, their efficiency remains almost constant independent of the size of the device itself, while the efficiency of other energy conversion processes scales dramatically with size. For this reason, in applications in which controlling the temperature of a small portion of a larger device (like for example in cooling down a $\mathrm{CPU}$ ) is fundamental, thermoelectric devices offer an economi$\mathrm{cal}$ and efficient solution. In the quest for efficiency, the properties of a wide range of materials have been investigated. Many have shown good thermoelectric efficiencies and this implies that in choosing the material for a certain application other parameters can be adjusted, for example, it is easily foreseen that silicon nanowires might found wider implementation in current electronic devices based on silicon. Opaque or transparent materials might find application in the field of photovoltaics, to increase the total current output of the device by using the part of the solar energy that is lost to waste heat.

Many strategies have been proposed to increase the figure of merit of a thermoelectric device: we can either increase $S, \sigma$, or the power factor $S^{2} \sigma$ or decrease the thermal conductance $\kappa$. It should become apparent that the maximisation of the figure of merit in bulk materials is difficult. Indeed, it is well known that the electrical and thermal conductances in bulk materials are not independent, but, for example in metals, they are con- nected by the Wiedemann-Franz empirical law 20 This law is based on the observation that in a metal, electrical and thermal currents are both carried by electrons. However, little is known about how to calculate the Seebeck coefficient. The definition of $S$ is quite generic,

$$
S=-\left.\frac{\Delta V}{\Delta T}\right|_{I=0}
$$

where $\Delta V$ is the voltage drop at the junctions if a temperature gradient $\Delta T$ is maintained, in an open circuit configuration, i.e., no electrical current is flowing. It has been suggested that a direct measure of this coefficient gives direct evidence of the sign of the carriers, a problem in complex nanoscale systems. ${ }^{21}$ This statement is generally true for bulk materials, but it has been pointed out that the theoretical approach used to understand the experimental results is based on the assumption that, at equilibrium, both the thermal and the electrical currents vanish. $\frac{22}{2}$ This assumption is not valid when we consider nanoscale systems, i.e., the ones for which we hope to get large figures of merit. It has been shown that removing this condition allows different signs of the Seebeck coefficient given the kind of carriers in the device while varying the working conditions. ${ }^{22}$

In this Perspective, I will discuss a nowadays standard approach to the calculation of the figure of merit, namely the combination of the Landauer's approach to quantum transport and the Onsager's theory of non-equilibrium response. I will propose alternative dynamical methods that can go beyond certain limitation of that standard approach. The paper is organised as follows. In the following section, we will discuss the Landauer's theory of quantum transport. We will show how one can arrive to simple expressions for the physical parameters entering the figure of merit $Z T$. In section 3 , we will discuss an alternative method for the calculation of the electrical conductance. In section 4, we will turn our attention to the Seebeck coefficient. Finally, in section 5 we will discuss the problem of evaluating the thermal conductance for electrons from a dynamical theory. In this latter case, we cannot provide an answer to this question, but we will discuss briefly two research lines which could lead to a comprehensive dynamical theory of the thermoelectric energy conversion. Throughout the paper we will set $\hbar=k_{B}=1$, where $k_{B}$ is the Boltzmann constant.

\section{Landauer's approach to quantum transport}

A widely used and successful approach to the calculation of the conductance in nano- and mesoscopic systems is the Landauer's formula. $\stackrel{18}{=}$ In this elegant and economic formalism one assumes that the details of the system are irrelevant far from

\footnotetext{
* Notice that the Wiedemann-Franz law could be formulated in terms of conductivities or conductances. For our scope, this distinction is irrelevant, since the same dimensional factors will multiply the numerator and denominator of 1
} 
the nanostructure in some region of space called reservoirs: the only property requested from these reservoirs is that their cross section be infinite, meaning that an electron entering that region cannot escape. A fixed bias keeps the two reservoirs polarized. The nanostructure is connected to the reservoirs through two regions of space which smoothly enter it. The transport properties are completely determined by the probability amplitude that an electron comes out of a reservoir, crosses the lead-nanostructure-lead device and is absorbed into the other reservoir. It is clear that this model contains many assumptions. We should be able to identify the different regions of interest (reservoir-lead-nanostructure) where different physics is at play, a separation lacking a solid justification from a microscopical point of view. ${ }^{23}$ Another basic assumption is that the many-particle states in the reservoirs are fully determined and do not mix; we are effectively neglecting particle-particle interaction. Finally the Landauer's approach cannot explain the nature of out-of-equilibrium states that are established in the device. In particular, it cannot answer the basic question, will such a system ever reach a steady state? Other approaches suffer from similar drawbacks. For example the non-equilibrium Green's function formalism (also known as the Keldysh formalism), $\underline{\underline{24}}$ although a powerful technique, assumes the existence of a steady state imposed by scattering boundary conditions far from the nano-system. The existence of a steady state seems a reasonable assumption for mesoscopic systems but needs to be proven for nanoscopic devices or closed system. ${ }^{16}$ Other adhoc models have been proposed in the past but they lack a clear physical interpretation. 18

Assuming the Landauer's approach can be applied, we start from the linear out-of-equilibrium Onsager's relations for the currents flowing along the device f

$$
\begin{aligned}
j & =L_{00} \Delta \mu+L_{01} \Delta T \\
j_{h} & =L_{10} \Delta \mu+L_{11} \Delta T .
\end{aligned}
$$

Symmetry considerations lead to $L_{01}=L_{10}$. Landauer's approach to thermal transport allows to express the Onsager's coefficients $L$ 's in terms of the transmission probability. In linear response with respect to the thermal gradient $\Delta T$ and the polarisation potential $\Delta \mu$, one obtains the general expressions

$$
L_{i j}(\mu, T)=\int_{-\infty}^{\infty} d E(E-\mu)^{i+j} \frac{\partial f(E, T, \mu)}{\partial E} \mathcal{T}(E) .
$$

In (5), the chemical potential $\mu=\left(\mu_{R}+\mu_{L}\right) / 2$, where $\mu_{R}$ and $\mu_{L}$ are the chemical potentials of the reservoirs, respectively. By assumption, $\mu_{L}-\mu_{R}=\Delta \mu \ll \mu_{L}, \mu_{R}$. The same is true for the temperature $T=\left(T_{R}+T_{L}\right) / 2$, with $\Delta T=T_{L}-T_{R} \ll$ $T_{R}, T_{L} . f(E, T, \mu)$ is the equilibrium Fermi distribution. We

$\dagger$ Here the particle and heat currents are assumed to be perpendicular to the cross section of the device at each position. We expect this to be correct for long nano-wires. assume indeed that the reservoirs are large and at equilibrium at given temperature and chemical potential. Moreover it is assumed that the electrons in the reservoir do not interact. This last assumption is justified by the fact that in metal the screening length is small. Finally, $\mathcal{T}(E)$ is the transmission probability for one electron with given energy $E$ to travel across the device. The theory needs to calculate this quantity to make the set of equations (4) and (5) of any use. We would like to point out here that while Landauer's approach to transport is valid beyond linear response, the Onsager's relations are valid only in linear response.

Given the Onsager's relations, and remembering the definition of the different physical quantities, we obtain the following relations between the microscopic parameters that enter the figure-of-merit and the integral $L$ 's,

$$
\begin{aligned}
\sigma & =e^{2} L_{00}(\mu, T), \\
S & =\frac{L_{10}(\mu, T)}{e T L_{00}(\mu, T)}, \\
\kappa_{e} & =\frac{1}{T}\left(L_{11}(\mu, T)-\frac{L_{10}^{2}(\mu, T)}{L_{00}(\mu, T)}\right) .
\end{aligned}
$$

In (6), $\kappa_{e}$ is the electronic contribution to the thermal conductance, defined by

$$
\kappa_{e} T=\left.\frac{j_{h}}{\Delta T}\right|_{j=0} .
$$

Here, we assume that we can write the total thermal conductance as $\kappa=\kappa_{e}+\kappa_{l}$, where $\kappa_{l}$ is the crystal (either phononic or vibronic) contribution to the thermal conductance. This is valid if the electron-phonon interaction is small. Only then we can separate these two contributions.

A step further into a complete theory of the thermoelectric energy conversion is the expression of the thermal conductance $\kappa_{l}$ in terms of the transmission probability of a phonon across the device,

$$
\kappa_{l}=\int_{0}^{\infty} d \omega \omega^{2} \frac{n(\omega, T)}{\partial T} \tau(\omega)
$$

where $n(\omega, T)$ is the Bose distribution of non-interacting phonon at given temperature $T$, and $\tau(\omega)$ is the probability that one phonon with energy $\omega$ could cross the device. $\stackrel{25-27}{-27}$

It is clear from (5), (6), and (8) that the only ingredients that we are still missing are the transmission probabilities $\mathcal{T}(E)$ and $\tau(\omega)$. A lot of progress has been made to connect these probabilities with the non-equilibrium Green's function theory and ultimately with electronic structure calculations. ${ }^{27}-30$ Remarkably, the transmission probability $\mathcal{T}$ can be written as

$$
\mathcal{T}(E)=\operatorname{Tr}\left[G^{r}(E) \Gamma_{L} G^{a}(E) \Gamma_{R}\right],
$$

where $G^{r}$ is the retarded Green's function, $G^{a}(E)=G^{r}(E)^{\dagger}$, and $\Gamma_{L(R)}=i\left(\Sigma_{L(R)}^{r}-\Sigma_{L(R)}^{a}\right)$ describe the coupling, via 
the self-energies $\Sigma^{r}$ and $\Sigma^{a}$ of the left and right reservoir with the device, respectively. Finally, the non-equilibrium Green's function is obtained from the Hamiltonian via,

$$
G^{r}(E)=\left[\left(E+i 0^{+}\right)+H_{C}-\Sigma_{L}^{r}-\Sigma_{R}^{r}\right]^{-1},
$$

where $H_{C}$ is the Hamiltonian of the central system. The Onsager's out-equilibrium response theory is exact in the linear approximation. Therefore, the quality of the results is strictly related to the approximations used to calculate the Hamiltonian $H_{C}$ and its eigenvalues. A certain degree of success has been obtained by using for the central region the Kohn-Sham (KS) Hamiltonian. $\stackrel{18,31,32}{ }$ To some extent, this degree of success is unexpected. The KS theory indeed, should not give access to the spectral properties of the system under investigation. Meanwhile, those properties are indeed strongly used in the evaluation of the non-equilibrium Green's functions. Finally, expressions similar to (9) and (10) can be written for the phonontransmission probability, with the substitution of $E$ with $\omega^{2}$, $H_{C}$ with $K$ the spring constant matrix, $G^{r}$ with $D^{r}$ the phonon non-equilibrium Green's function.

The fact that one can tackle on the same ground both the vibrational and electronic contribution to the figure-of-merit is one of the greatest advantages of this theory. Moreover, its simplicity in expressing all the quantities of interest in terms of the transmission probability and this one in term of a microscopic model of the device, is invaluable in making the theory predictive. $\underline{33}$

At the same time, to gain a deeper understanding on the physics of thermoelectric energy conversion, we should be able to build other models and theories that can extend or confirm the validity of the Landauer/Onsager's approach to the calculation of the figure-of-merit. Over the years, many novel approaches have been proposed which are trying to go beyond the actual state-of-the-art in molecular transport, by, e.g., implementing a $G W$ approximation for the calculation of the nonequilibrium Green's function and apply it to transport in molecular devices ${ }^{34,35}$ or by building a suitable base for calculating the time evolution of the electrons. $\frac{36}{}$ In the following we will discuss other ways, strongly based on Time Dependent Density Functional Theory (TDDFT), $\underline{10}$ to calculate the Seebeck coefficient and the electrical conductance by using dynamical theories. Here, one studies the dynamics of the electrons as they respond to the external perturbation that generates the electrical and heat flows. In general, these theories have the possibility to go beyond the linear response, and assess non-linear effects. The drawback is that the computation of the electron dynamics is more expensive than any static calculation. At the same time, the dynamical theories are still in their infancy. This means that we do not have reliable approximations to the many-body Hamiltonian that allow a correct evaluation of the dynamics of the observable, although some remarkable progress has been made through, for example, exact solutions ${ }^{37}$ or in correlating the dynamics of the electrons with the ions. .38 .39 In this respect, further investigation is necessary. While these alternative models do allow the evaluation of the Seebeck coefficient and the electrical conductance, little can be said about the thermal conductances, especially for the electrons. In Section 5, we will shortly address this question and outline two possible research paths for modelling the thermal electronic conductance with dynamical theories.

\section{Time-dependent (current-)density functional theory}

Density Functional Theory (DFT) has become a ubiquitous tool in the investigation of the properties of matter. ${ }^{31,32}$ The reasons for this success are manifold and introducing the reader to the field is well outside the scope of this article. It will suffice to say that DFT gives in principle access to an exact solution of the many-body problem, whenever we can focus only on certain quantities, like, e.g., the single particle density. In its original formulation, DFT deals with the problem of calculating the ground state energy and single particle density of a many-body electronic system. Over time, many extensions of the original idea have been presented: the most interesting for our discussion are the TDDFT and the Time-Dependent Current-Density Functional Theory (TDCDFT). ${ }^{10,32,40,41}$ As their names suggest, the two theories deal with the problem of calculating the dynamics of a many-body system. The result is obtained by mapping the dynamics of the real system onto the dynamics of a fictitious many-body system of non-interacting particles. In the case of TDDFT, we are interested in the single-particle density, $n(r, t)$. Interestingly, TDDFT does not give access to the current density $j(r, t)^{\underline{42}}$ but it can reproduce the exact total current. ${ }^{43}$ On the other hand, TDCDFT gives access to the exact single-particle current density, and via the continuity equation, to the single-particle density. In this respect TDCDFT is a more general theory than TDDFT. We would like to point out that DFT and TDDFT should be able to deal with the dynamics of strongly correlated systems. In this direction, remarkable results have been obtained in the solution of the Kondo problem, see for example 44 and references therein. One of the advantages of this theory over the standard non-equilibrium Green's function formalism is that it avoids the separation of the system intro three (or more) parts. In general, indeed for the nonequilibrium Green's function we need to identify the left and right reservoirs and connect them with the central region. In most of the cases, this procedure lacks a clear physical interpretation ${ }^{23}$ and may produce spurious effects. On the other hand, in TDCDFT one can investigate the full dynamics of the system without reverting necessarily to this separation. $\frac{13,45,46}{}$

It should also be clear that TDCDFT is built to treat problems where electrical transport is important. By reproducing 
the single-particle current for given external potential, the electrical conductance, $\hat{\sigma}$ can be obtained directly from its microscopical definition

$$
\vec{j}(r, t)=\int d r^{\prime} \hat{\sigma}\left(r, r^{\prime}\right) \cdot \vec{E}\left(r^{\prime}, t\right) .
$$

Since the electrical field $\vec{E}(r, t)$, is an external input, (11) allows the complete determination of the conductance tensor, if the current density can be calculated. Moreover, the theory does not suffer from any problem of dividing the device/reservoir region. In principle, we can treat the motion of the electrons exactly in the presence of an external polarizing potential. ${ }^{15}$

\section{Calculation of the Seebeck coefficient}

We will now turn our attention to the calculation of the Seebeck coefficient. It should be apparent from its definition (3) that the calculation and measurement of the Seebeck coefficient are a difficult task. Indeed, in the experiments we combine the ability to control the temperature gradient with the measurement of small bias drops while maintaining an open circuit configuration. These technical difficulties reflect into a large uncertainty in the values of the Seebeck coefficient which is usually the result of indirect measurements which in turn require a good level of theoretical understanding. From a theoretical point of view is then of the uttermost interest to have solid and reliable ways to calculate the Seebeck coefficient for many different materials. In this respect, the theory we have exposed in Section 2 has proven an invaluable tool. Nonetheless, we must find an alternative formulation that will teach us the strengths and weaknesses of the Landauer's approach and provide novel tools to pursue our quest of efficient thermoelectric materials. Ideally, we would like to revert to the basic definition of the Seebeck coefficient, $S=-\Delta V / \Delta T$ at vanishing electrical current. $\Delta V$ is the response of the system to the external thermal gradient, $\Delta T$. We then need a theory able to describe the dynamics and steady state of a quantum mechanical system coupled to two external baths, or, more in general, in contact with an external environment.

The theory of open quantum systems has been devised with this aim in mind. ${ }^{47-53}$ The general starting point is the Hamiltonian of the composite system: device plus external environment. Generally we can write that Hamiltonian as

$$
H=H_{C}+H_{E}+H_{\text {coup }}
$$

where $H_{C}$ is the Hamiltonian of the isolated system, $H_{E}$ the Hamiltonian of the environment, and $H_{\text {coup }}$ describes the coupling between the two. The aim of the theory is to "fold" the degrees of freedom of the environment and obtain an effective equation of motion for the system dynamics. If we assume the coupling has the form

$$
H_{\text {coup }}=\lambda V \otimes B,
$$

where $V$ and $B$ are system and environment operators, respectively, and $\lambda$ is a small coupling parameter, a standard procedure leads to the equation of motion for the "state' $|\Psi\rangle$ of the system $25.54-56$

$$
\begin{aligned}
i \frac{\partial}{\partial t}|\Psi(t)\rangle= & -i H_{C}|\Psi(t)\rangle \\
& +\lambda^{2} \int_{0}^{t} d \tau C(\tau) V^{\dagger}(\tau) e^{-i H_{C} \tau} V|\Psi(\tau-t)\rangle \\
& +\lambda \eta(t) V|\Psi(t)\rangle,
\end{aligned}
$$

where $C(t)=\operatorname{Tr}_{E}\left[\rho_{E}^{e q} B(t) B^{\dagger}(0)\right]$ is the environment correlation function. We assume that the environment is maintained in the thermal equilibrium described by the statistical operator

$$
\rho_{E}^{e q}=\frac{e^{-\beta H_{E}}}{\operatorname{Tr}_{E} e^{-\beta H_{E}}}
$$

and $\eta(t)$ is a complex coloured noise with the statistical properties 52

$$
\overline{\eta(t)}=0, \overline{\eta(t) \eta(0)}=0, \overline{\eta(t) \eta^{*}(0)}=C(t) .
$$

Here with the $\cdots$ we indicate the average taken after many realisations of the noise.

The stochastic Schrödinger equation (SSE) (14) is able to reproduce the dynamics of the density matrix for the system, $, 54,55,57,58$

$$
\rho=\frac{\overline{|\Psi\rangle\langle\Psi|}}{\overline{\langle\Psi \mid \Psi\rangle}} .
$$

Interestingly, it is the average over the many realisations of the noise that makes this density matrix not pure.

A standard approximation consists in replacing the correlation function $C(t)$ with a Dirac $\delta(t)$ function. This corresponds to neglecting the time correlation of the bath, effectively assuming the environment has no memory - a Markov approximation. Within the Markov approximation, the SSE assumes the simplified form

$$
\begin{aligned}
i \frac{\partial}{\partial t}|\Psi(t)\rangle= & -i H_{C}|\Psi(t)\rangle \\
& +\lambda^{2} V^{\dagger} V|\Psi(t)\rangle+\lambda \eta(t) V|\Psi(t)\rangle .
\end{aligned}
$$

Using the definition, we can derive a simple form for the dynamics of the master equation,

$$
\frac{d \rho}{d t}=-i[H, \rho]-V \rho V^{\dagger}+\frac{1}{2} V^{\dagger} V \rho+\frac{1}{2} \rho V^{\dagger} V .
$$

$\ddagger$ It is important to point out that the state $\Psi$ generally does not describe a true quantum trajectory. 54 
It can be proven that this is the most general memoryless master equation valid up to second order in $\lambda$ that preserves the positivity of the density matrix. $\underline{59}$

The equations (18) and (19) can be easily generalised to the case of the system being in contact with more than one bath operator $B$. In doing so, we can assume that each of these baths corresponds to a different thermal equilibrium (for example with different temperatures) and then study the dynamics of the system under this now out-of-equilibrium conditions. This idea has been explored partially to investigate the thermoelectric energy conversion with (19).22 It is important to point out that in principle, in the Markov approximation, the detailed balance equation that will ensure the establishment of thermal equilibrium when the temperatures of the baths are the same, is not fulfilled. ${ }^{54}$ Moreover, it is not clear how to treat particleparticle interaction in this formalism, since that in general will make the Hamiltonian state-dependent. The derivation of the master equation in this case appears more subtle and care is needed to avoid spurious effects. $\stackrel{60}{=}$ For this reason, a TDCDFT scheme for this open quantum system has been recently developed that can go beyond the limits of the density matrix formalism. $\stackrel{60,61}{ }$ This scheme, by coupling the strength of a DFT approach with the theory of open quantum systems, could help in establishing a framework for the investigation of the Seebeck coefficient of materials. Indeed, being able to couple the device of interest with two baths at different temperatures, we can study the formation of the potential difference $\Delta V$ in time, by using the Poisson equation, since the electrons in the system will try to oppose to the thermal gradient by piling up closer to the coolest bath. At equilibrium, no electronic macroscopic current will be present in the device, and we can use the definition (3) to evaluate the Seebeck coefficient. ${ }^{22}$ The same theory can be used to investigate the validity of the Fourier law at the nanoscale. .62

\section{Thermal conductance of electrons}

As we have seen in the evaluation of the figure of merit, the Seebeck coefficient and the electrical conductance can be calculated by using dynamical methods thus offering an alternative description to the Landauer formalism.

The situation is less clear with respect to the calculation of the thermal conductance. In this case, there is not a dynamical formulation of the problem in terms of a density functional approach. The reason for this situation is simple: The energy current $j_{h}$ cannot be written in terms of the electrical (or density) current. Indeed, we can define the energy current across a given section of the system as

$$
j_{h}=-\frac{d}{d t}\left\langle H_{S}\right\rangle
$$

where $H_{S}$ is the energy stored in the volume enclosed by the surface we are considering. For example, if we can separate the system into left, right and central region, where the left and right act as energy "reservoirs", $H_{S}$ is the energy stored in one of these two reservoirs. We could then write a similar equation for the energy coming from the other reservoir. Combining the two, we can calculate the energy flow in the system. A naive approach would simply suggest to calculate the energy starting from the DFT wavefunctions, in a fashion similar to what has been done in the past to evaluate the non-equilibrium Green's function. However, this operation contains a certain number of approximations, many of which are not under control. Indeed, the theorem of DFT (either the static or dynamical) cannot give access to the energy of the excited states. For this reason, the concept of the total energy stored in a part of the system will bear little meaning in a DFT formulation of the problem.

We foresee two ways out of this impasse. On the one hand, one can think of building an ad-hoc density functional theory that provides the exact energy or thermal current. To do so, we need to identify a suitable potential that is connected to the energy current, responsible to generate that current in the many-body system. If that potential is found, its existence is doubtful to say the least, then we can hopefully prove that the mapping between the potential and the energy current is oneto-one. If this can be done, and a suitable KS scheme be built, we will have an exact formulation of the problem in terms of non-interacting particles and we can compute the energy or the thermal current from first principles.

However, one can build some approximate model to the electron thermal transport. In this respect a hydrodynamical formulation of the electronic transport, would provide a set of equations which includes the thermal conductance and the specific heat of the electron liquid. See for example Ref. $\frac{12}{}$ for the formulation of the theory for a quantum point contact. In Ref. 12 the hydrodynamical equations were used to calculate the electronic temperature in the nanojunction. However, if we do assume that temperature can be either modelled or measured independently, these equations will be sufficient to calculate the thermal conductance of the electrons. An ab-initio theory here will enter in defining the viscous coefficient of the electron liquid.

In a hydrodynamic approach to quantum transport, we define the stress tensor starting from the velocity of the fluid, $\vec{v}(r, t)=\vec{j}(r, t) / n(r, t)$,

$$
\pi_{i, j}=\eta\left(\nabla_{i} v_{j}+\nabla_{j} v_{i}-\frac{2}{3} \delta_{i, j} \nabla_{k} v_{k}\right)
$$

where $\eta$ is a real coefficient (the viscosity) that is a functional of the density. $12,63,64$ We point out that (21) is in fact a particular case of a general stress tensor with memory effects taken into account. .65 The parameters of the liquid can be build from a microscopic theory of the low-energy excitations of the system under investigation. Unfortunately, it turns out that their 
detailed dependence on the geometry and dimensionality of the system is complex. ${ }^{64}$ It is clear that further investigation in this direction is needed. In terms of this stress tensor, the continuity equation and the equation of motion for the velocity field are written as,

$$
\begin{aligned}
D_{t} n(r, t)= & -n(r, t) \nabla \cdot \vec{v}(r, t), \\
m n(r, t) D_{t} v_{i}(r, t)= & -\nabla_{i} P(r, t)+\nabla_{j} \pi_{i, j}(r, t) \\
& -n(r, t) \nabla_{i} V_{e x t}(r, t) .
\end{aligned}
$$

where $D_{t}=\partial / \partial_{t}+v(r, t) \cdot \nabla$ is the convective derivative, $m$ the electron mass, $P(r, t)$ is the pressure, and $V_{\text {ext }}(r, t)$ is a timedependent external potential. The second equation is nothing else than the Navier-Stokes equation for the a viscous fluid. 12,68 It is interesting to point out that for a nanoscale system, for electronic low-energy excitations, this theory is equivalent to the many-body formulation.

To study the thermal transport, we need to complement (22) with an equation for the energy flow. For a quantum point contact, a nanoscale constriction between two large metallic reservoirs, one obtains, 12

$$
\pi_{i, j}(r) \partial_{j} v_{i}(r)+\nabla \cdot\left[k(r) \nabla T_{e}(r)\right]=c_{V}\left(T_{e}\right) \vec{v}(r) \cdot \nabla T_{e}(r),
$$

where $T_{e}$ is the electronic temperature, $k(r)$ is the diffusion constant and $c_{V}$ is the specific heat at fixed volume of the electron gas. Obviously, in writing Eq. (23) we have assumed that some thermodynamic quantities like temperature and entropy for an electron liquid flowing in a nanostructure can be defined. This is a much debated point, and obviously we do not have a general solution for it. However, here we argue that the electron temperature may be defined as the one ideally measured by a probe weakly coupled to the system and in local equilibrium with the latter. $\frac{18,22}{}$ The set of hydrodynamical equations has been used to study the thermal heating of a nanoscale device. They do predict novel and interesting phenomena, like a cooling of the phonon modes due to the electron heating. 12 This result has been beautifully confirmed by experience. ${ }^{69}$

I am personally amazed that the equation of motion for a many-body system does reduce to a form similar to the NavierStokes equations of a classical liquid. The quantum mechanical effects are still embodied by the pressure term $P(r)$. For this reason, the solution of these Navier-Stokes equations is somehow more difficult than the already demanding solution of the classical equations. Finally, it is quite surprising that TDCDFT arrived at this same conclusion well before. 55,66 At the same time, TDCDFT seems to suggest natural mechanisms for energy relaxation, ${ }^{70}$ and recently a thermodynamical interpretation of the KS energy has been given, as the maximal work that can be extracted from the system. ${ }^{71}$ Starting from these considerations, it does appear possible that TDCDFT can be used to describe some of the physical process related to energy dissipation and transfer.
Both formulations of the problem of calculating the electron thermal transport will require the solution of long-standing problems. For example, in a density functional theory for the thermal current, we will very soon discover that a thermal current is generated by almost any external potential, not only by a temperature imbalance. Therefore, how can a given thermal current be in a one-to-one mapping with a certain potential? The hydrodynamical formulation can be equally as challenging. For example, what is the range of validity of the equations of motion. For sure, at short wavelengths, the electrons do not behave as a liquid. Moreover, at the nanoscale, a few very fundamental equations of classical heat transport, like the Fourier equation, are not valid. The analysis of thermal transport at the nanoscale thus should include a wider analysis on the validity of assumptions made on the dynamics of the system.

We have left out the problem of phonon/vibron thermal transport, and the coupling between the electrons and the vibrons. Presently, besides doing a full dynamics of the coupled electronic and atomic motions, we do not see an alternative to the static calculations performed starting from (8), and improving the calculation of the Green's function via perturbation theory. A lot of effort has been put into the calculation of the phonon thermal conductance, for example in silicon based nano-structures, ${ }^{72,73}$ via either molecular dynamics or the Boltzmann transport equation. While these techniques have been proven useful in calculating the thermal conductance, it also remains difficult to obtain full convergence of the dynamics especially when the system is maintained out of equilibrium. On the other hand, it is true that (8) is limited to the harmonic approximation for the phonon spectrum. This approximation appears to be valid only for low energy vibrationssince the interaction parameter vanishes with vanishing phonon energy-which are also responsible for the largest contribution to thermal transport.

\section{Conclusions}

The investigation of the thermoelectric properties of materials poses interesting challenges because it does require the treatment, on equal footing, of electrical and thermal transport. So far the only theory that has succeeded in doing so is the linear response theory developed by using the Onsager's relations coupled with the Landauer's approach to thermal transport. While successful for many systems, it is necessary to have alternative theories able to describe the same systems and go beyond the non-equilibrium linear response regime. In this article, I have discussed the state-of-the-art of one of the alternatives, namely a dynamical approach to the calculation of the thermoelectric coefficients. While the theory is based on solid ground for the Seebeck coefficient and the electrical conductance, the thermal conductance, both for the electronic and atomic systems, is more difficult to access with first principle theories. I have 
sketched a couple of possible research routes that could give access to this important piece of information.

\section{Acknowledgments}

The author acknowledges support from MICINN (FIS2010-21282 C02-01 and PIB2010US-00652), Grupos Consolidados UPV/EHU del Gobierno Vasco (IT-319-07), and ACI-Promociona (ACI2009-24 1036), the financial support of the CONSOLIDER-INGENIO 2010 "NanoTherm" (CSD2010-00044) of the MICINN and is thankful for its hospitality to the Physics Department of the King's College London.

\section{References}

1 F. J. DiSalvo, Science, 1999, 285, 703-706.

2 C. B. Vining, Nature Mat., 2008, 7, 765.

3 M. S. Dresselhaus, G. Chen, M. Y. Tang, R. Yang, H. Lee, D. Wang, Z. Ren J.-P. Fleurial and P. Gogna, Adv. Mater, 2007, 19, 1043.

4 D. Pollock, Thermoelectricity; theory, thermometry, tool, ASTM, Philadelphia, 1985.

5 H. J. Goldsmid, Introduction to Thermoelectricity, Springer-Verlag, Berlin, 1st edn., 2010, p. 250

6 D. L. Klein, R. Roth, A. K. L. Lim, A. P. Alivisatos and P. L. McEuen, Nature, 1997, 389, 699 .

7 S. Kubatkin, A. Danilov, M. Hjort, J. Cornil, J.-L. Brédas, N. StuhrHansen, P. Hedegå rd and T. Bjø rnholm, Nature, 2003, 425, 698.

8 Introducing Molecular Electronics, ed. G. Cuniberti, G. Fagas and K. Richter, Springer, New York, 2005.

9 Introduction to Nanoscale Science and Technology, ed. M. Di Ventra S. Evoy and J. R. Heflin, Springer, New York, 2004.

10 Time-Dependent Density Functional Theory, ed. M. A. L. Marques, C. A. Ullrich, A. Rubio, F. Nogueira, K. Burke and E. K. U. Gross, Springer, Berlin, 2006, vol. 706.

11 Y.-C. Chen, M. Zwolak and M. Di Ventra, Nano Lett., 2003, 3, 1961

12 R. D’Agosta, N. Sai and M. Di Ventra, Nano Lett., 2006, 6, 2935-8.

13 N. Bushong, N. Sai and M. Di Ventra, Nano Lett., 2005, 5, 2569.

14 N. Sai, N. Bushong, R. Hatcher and M. Di Ventra, Phys. Rev. B, 2007, 75 115410 .

15 G. Stefanucci and C.-O. Almbladh, Phys. Rev. B, 2004, 69, 195318.

16 G. Stefanucci, Phys. Rev. B, 2007, 75, 195115.

17 C. Vining, Nature Mat., 2009, 8, 83-85.

18 M. Di Ventra, Electrical transport in nanoscale systems, Cambridge University Press, New York, 1st edn., 2008.

19 L. D. Hicks and M. S. Dresselhaus, Phys. Rev. B, 1993, 47, 12727-12731.
20 N. W. Ashcroft and N. D. Mermin, Solid State Physics, Saunders College Publishing, 1976.

21 P. Reddy, S. Y. Jang, R. A. Segalman and A. Majumdar, Science, 2007, 315, 1568.

22 Y. Dubi and M. Di Ventra, Nano Lett., 2009, 9, 97-101.
L. P. Kadanoff and G. Baym, Quantum Statistical Mechanics, Westview Press, New York, 1962.

25 M. P. Blencowe, Phys. Rev. B, 1999, 59, 4992-4998.

26 M. P. Blencowe, Phys. Rep., 2004, 395, 159-222.

27 T. Yamamoto and K. Watanabe, Phys, Rev. Lett., 2006, 96, 255503.

28 Y. Meir and N. Wingreen, Phys. Rev. Lett., 1992, 68, 2512-2515.

29 A.-P. Jauho, N. S. Wingreen and Y. Meir, Phys. Rev. B, 1994, 50, 5528.

30 N. Mingo, Phys. Rev. B, 2006, 74, 125402.

31 W. Kohn and L. J. Sham, Phys. Rev., 1965, 140, A1133-A1138.

32 G. F. Giuliani and G. Vignale, Quantum Theory of the Electron Liquid, Cambridge, Cambridge, UK, 1st edn., 2005.

33 S. Y. Quek, H. J. Choi, S. G. Louie and J. B. Neaton, ACS nano, 2011, 5, 551.

34 K. S. Thygesen, Phys. Rev. Lett., 2008, 100, 166804.

35 K. Thygesen and A. Rubio, Phys. Rev. B, 2008, 77, 115333.

36 P. Bokes, J. Jung and R. Godby, Phys. Rev. B, 2007, 76, 125433.

37 J. Maciejko, J. Wang and H. Guo, Phys. Rev. B, 2006, 74, 085324.

38 N. Sergueev, D. Roubtsov and H. Guo, Phys. Rev. Lett., 2005, 95, 146803.

39 J.-T. Lü, M. Brandbyge, P. Hedegå rd, T. Todorov and D. Dundas, Phys. Rev. $B, 2012, \mathbf{8 5}, 245444$.

40 E. Runge and E. K. U. Gross, Phys. Rev. Lett., 1984, 52, 997-1000.

41 S. Ghosh and A. Dhara, Phys. Rev. A, 1988, 38, 1149-1158.

42 R. D’Agosta and G. Vignale, Phys. Rev. B, 2005, 71, 245103.

43 M. Di Ventra and T. N. Todorov, J. Phys.: Cond. Matt., 2004, 16, 8025.

44 G. Stefanucci and S. Kurth, Phys. Rev. Lett., 2011, 107, 216401.

45 G. Stefanucci, S. Kurth, A. Rubio and E. K. U. Gross, Phys. Rev. B, 2008, 77, 75339.

46 S. Kurth, G. Stefanucci, C.-O. Almbladh, A. Rubio and E. K. U. Gross, Phys. Rev. B, 2005, 72, 035308.

47 R. Feynman and F. L. Vernon, Ann. Phys., 1963, 24, 118.

48 N. Gisin, Journal of Physics A: Mathematical and Theoretical, 1981, 14, 2259.

49 A. O. Caldeira and A. J. Legget, Physica A, 1983, 121, 587.

50 L. Diósi and W. T. Strunz, Phys. Lett. A, 1997, 235, 569. 
51 W. Strunz, Phys. Rev. A, 1996, 54, 2664-2674.

52 C. W. Gardiner and P. Zoeller, Quantum Noise, Springer, Berlin, 2nd edn., 2000, p. 438.

53 U. Weiss, Quantum Dissipative Systems, World Scientific, Singapore, 3rd edn., 2007.

54 R. Biele and R. D'Agosta, J. Phys.: Cond. Matt., 2012, 24, 273201.

55 P. Gaspard and M. Nagaoka, J. Chem. Phys., 1999, 111, 5676.

56 N. G. van Kampen, Stochastic Processes in Physics and Chemistry, Elsevier, Amsterdam, 3rd edn., 2007.

57 W. T. Strunz, L. Diósi and N. Gisin, Lecture Notes in Physics, 2000, 538, 271-280.

58 I. de Vega, D. Alonso and P. Gaspard, Phys. Rev. A, 2005, 71, 023812.

59 G. Lindblad, Communications in Mathematical Physics, 1976, 48, 119130 .

60 R. D’Agosta and M. Di Ventra, Phys. Rev. B, 2008, 78, 165105.

61 M. Di Ventra and R. D’Agosta, Phys. Rev. Lett., 2007, 98, 226403.

62 Y. Dubi and M. Di Ventra, Rev. Mod. Phys., 2011, 83, 131.

63 I. V. Tokatly and O. Pankratov, Phys. Rev. B, 1999, 60, 15550.

64 R. DAgosta, M. Di Ventra and G. Vignale, Phys. Rev. B, 2007, 76, 035320.

65 S. Conti and G. Vignale, Phys. Rev. B, 1999, 60, 7966-7980.

66 G. Vignale and W. Kohn, in Electronic density functional Theory: recent progress and new directions, ed. J. F. Dobson, G. Vignale and M. P. Das, Plenum, N.Y., 1996, p. 199.

67 I. V. Tokatly, Phys. Rev. B, 2005, 71, 165105.

68 L. D. Landau and E. M. Lifshitz, Fluid mechanics, Pergamon Press Ltd., 2nd edn., 1987, vol. 6.

69 Z. Huang, F. Chen, R. D’Agosta, P. A. Bennett, M. Di Ventra and N. Tao, Nature Nanotech., 2007, 2, 698-703.

70 H. O. Wijewardane and C. A. Ullrich, Phys. Rev. Lett., 2005, 95, 86401.

71 R. D’Agosta and G. Vignale, Phys. Rev. Lett., 2006, 96, 016405.

72 D. Donadio and G. Galli, Phys. Rev. Lett., 2009, 102, 195901.

73 D. Donadio and G. Galli, Nano Lett., 2010, 10, 847-851. 